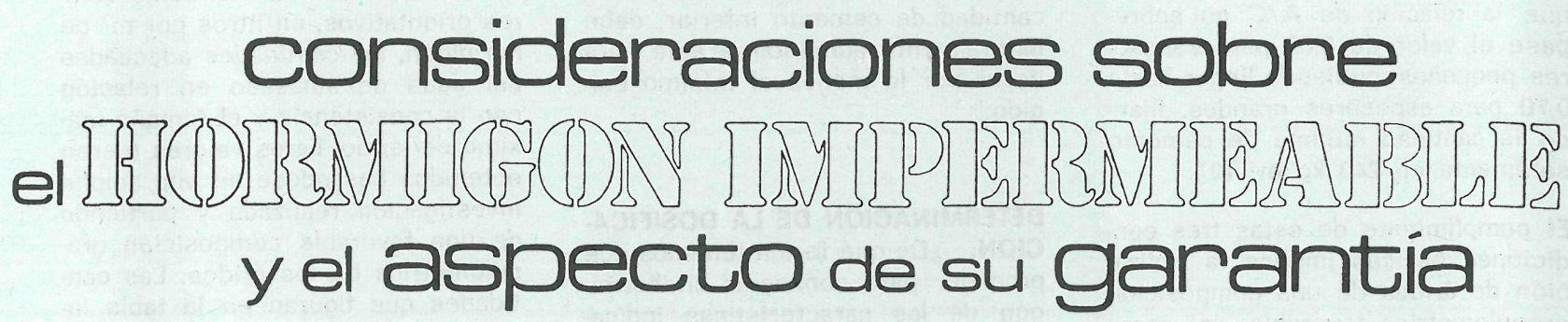

CARLOS SAFRANEZ, Dr. ingeniero

\title{
sinopsis
}

INTRODUCCION. Al proyectar la impermeabilización de un depósito de agua, de una obra subterránea como un sótano, un túnel, etc., disponemos de dos procedimientos diferentes para lograr la impermeabilidad requerida: El primero consiste en recubrir la superficie del hormigón con una capa impermeable para impedir la penetración del agua dentro de la estructura; el segundo reside en hacer impermeable toda la masa del hormigón, o sea, la impermeabilización integral del mismo.

Al decidirse a emplear este último procedimiento, es de suma importancia aclarar y fijar las características necesarlas que tiene que reunir el hormigón fresco para que, una vez fraguado, resulte impermeable o, mejor dicho, lo más impermeable posible.

No es correcto hablar de la impermeabilidad del hormigón en términos absolutos, ya que su impermeabilidad efectiva no depende solamente de su calidad, sino también de su espesor, de la presión del agua y del tiempo de su actuación, dejando aparte las características de la estructura.

Sabemos que el exigir el empleo de un aditivo impermeabilizante no es suficiente para asegurar la impermeabilidad del hormigón. Según la definición de las Normas alemanas de aditivos para el hormigón, la acción del aditivo impermeabilizante consiste en aumentar la resistencia del hormigón a la absorción del agua y a su penetración dentro de la masa del hormigón (1).

Por muy eficiente que sea un impermeabilizante, no es, naturalmente, capaz de convertir un hormigón de mala calidad en un hormigón bueno. Lo que se pretende al emplear un aditivo impermeabilizante es convertir un hormigón de buena calidad, pero no lo suficientemente impermeable, en un hormigón impermeable de acuerdo con las exigencias de la obra.
También sabemos que la cantidad de cemento a emplear, por sí sola, tampoco define la calidad del hormigón. Lo que tiene en cambio una importancia decisiva, es la relación agua/cemento $(A / C)$, y la composición granulométrica de los áridos.

CONDICIONES BASICAS DEL HORMIGON IMPERMEABLE. Las características que tiene que reunir el hormigón para que resulte impermeable pueden resumirse en las tres condiciones esenciales siguientes:

1. El hormigón fresco, empleando la cantidad mínima de agua de amasado, debe tener la docilidad necesaria para asegurar su buena compactación.

2. El hormigón fraguado debe tener una estructura muy homogénea y sin huecos.

3. La relación $\mathrm{A} / \mathrm{C}$ no debe rebasar un determinado valor, empleando una cierta cantidad mínima de cemento por $\mathrm{m}^{3}$. 
En Alemania p. e. se recomienda que la relación de $A / C$ no sobrepase el valor de 0,60 para espesores pequeños, pudiendo llegar hasta 0,70 para espesores grandes, fijando la cantidad mínima de cemento solamente en $240 \mathrm{~kg} / \mathrm{m}^{3}$ (2).

El cumplimiento de estas tres condiciones básicas implica la utilización de áridos de una composición granulométrica favorable, así como también el empleo de aditivos destinados a modificar las propiedades del hormigón con el fin de adaptarlo mejor a la obra a ejecutar. Más adelante nos ocuparemos detalladamente de estos requisitos.

En la «Instrucción" del Ministerio de Obras Públicas para el proyecto y la ejecución de obras de hormigón se exige para el hormigón armado la dosificación mínima de cemento de $250 \mathrm{~kg} / \mathrm{m}^{3}$, sin mencionar específicamente al hormigón impermeable, pero se entiende que esta cantidad no debe rebasarse tampoco para este último. La cantidad máxima de cemento por $\mathrm{m}^{3}$ de hormigón no debe ser superior a $400 \mathrm{~kg}$ debido al peligro de la retracción del fraguado (3).

Al ocuparnos de la dosificación del hormigón, veremos que al calcular la cantidad necesaria de cemento para tener un hormigón impermeable se obtiene normalmente una cantidad por encima de los $250 \mathrm{ki}$ logramos $/ \mathrm{m}^{3}$. En el caso de que el cálculo nos dé como resultado una cantidad de cemento inferior, debe naturalmente aumentarse ésta para llegar por to menos al mínimo exigido.

DETERMINACION DE LA DOSIFICACION. ¿De qué forma tenemos que proceder para conseguir un hormigón de las características indicadas? Tenemos que partir, naturalmente, de la composición del hormigón fresco, que se compone de cemento, áridos, agua y aditivos, aparte de las burbujas de aire ocluidas en la mezcla.

Para determinar la dosificación adecuada tenemos que tener muy en cuenta la interrelación que existe entre los distintos componentes de la mezcla, por lo que es muy recomendable tomar como punto de partida la cantidad necesaria del agua de amasado.

CANTIDAD DE AGUA. Esta cantidad depende de los tres siguientes factores:

1. De la composición granulométrica de los áridos.

2. Del tamaño máximo de su grano.

3. De la consistencia que debe tener la mezcla.
En la tabla 1 se reseñan como valo. res orientativos, en litros por $\mathrm{m}^{3}$ de hormigón, las cantidades adecuadas del agua de amasado en relación con la consistencia y el tamaño máximo del árido. Estos valores fueron obtenidos basándose en una amplia investigación realizada y partiendo de una favorable composición granulométrica de los áridos. Las cantidades que figuran en la tabla incluyen la humedad propia de los áridos, pero sin tener en cuenta la posible reducción de agua debido al empleo de los aditivos, lo que supone normalmente del 5 al $15 \%$ (2). Más adelante y al tratar de los aditivos volveremos sobre este efecto.

El contenido en finos, o sea, el conjunto del cemento y de los áridos hasta $0,2 \mathrm{~mm}$, tiene también una influencia sobre la cantidad del agua necesaria, aumentando ésta al aumentar la cantidad de finos. La tabla 1 está basada en la cantidad fija de $350 \mathrm{~kg} / \mathrm{m}^{3}$ en finos, pero al sobrepasar este valor hay que aumentar la cantidad del agua en un $10 \%$ sobre el exceso de los finos:

Aumento del agua:

$$
A a=0,1(F-350) \text { litros } / m^{3} ;
$$

en la cual:

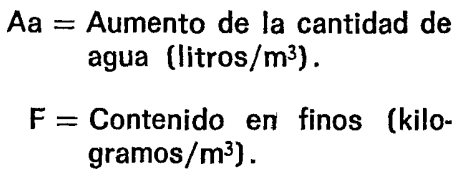

\begin{tabular}{|c|c|c|c|c|c|}
\hline \multirow{3}{*}{ CONSISTENCIA } & \multicolumn{5}{|c|}{ TAMANO MAXIMO DEL ARIDO } \\
\hline & $7 \mathrm{~mm}$ & $15 \mathrm{~mm}$ & $30 \mathrm{~mm}$ & $50 \mathrm{~mm}$ & $70 \mathrm{~mm}$ \\
\hline & \multicolumn{5}{|c|}{ Cantidad total del agua de amasado $\mathrm{Ag}$ en litros $/ \mathrm{m}^{3}$ para hormigones $\sin$ aditivos } \\
\hline C-1 & $200-220$ & $175-200$ & $150-175$ & $140-165$ & $135-160$ \\
\hline $\mathrm{C}-2$ & $210-230$ & $185-210$ & $160-185$ & $150-175$ & $140-170$ \\
\hline C-3 & $225-250$ & $195-220$ & $\therefore 170-200$ & $160-185$ & $155-180$ \\
\hline C-4 & $240-270$ & $215-240$ & $185-215$ & $175-205$ & $170-195$ \\
\hline
\end{tabular}

TABLA 1. Valores orientativos del agua de amasado 
Más adelante volveremos igualmente sobre el contenido en finos.

Las consistencias indicadas en la tabla 1 se valoran del modo siguiente:

CONSISTENCIA C-1. Hormigón muy seco. Límite superior de la posibilidad de su compactación por medio de vibradores potentes. La parte del mortero fino de la mezcla está solamente un poco más húmeda que una tierra húmeda. El hormigón, al verter, forma una masa suelta no coherente.

CONSISTENCIA C-2. Hormigón entre seco y plástico, fácilmente compactable por medio de vibradores, pero límite superior de la posibilidad para picar con barra. La parte del mortero fino de la mezcla es de consistencia pastosa-mantecosa. El hormigón, al verter, forma trozos coherentes.

CONSISTENCIA C-3. Hormigón plástico. Límite inferior de la posibilidad de su compactación por medio de vibradores ligeros. Se pica con barra fácilmente. Se emplea para espesores pequeños y también en las partes de la estructura donde la armadura resulta muy tupida. La parte del mortero fino de la mezcla forma una pasta blanda. El hormigón, al verter, resulta coherente y blando. El escurrimiento, según DIN 1048 , es de unos $36 \mathrm{~cm}$, con un máxìmo de $40 \mathrm{~cm}$.

CONSISTENCIA C-4. Hormigón fluido. No es necesaria mucha compactación. La parte fina del mortero de la mezcla resulta fluida. El hormigón, al verter, es blando y fluido. El escurrimiento, según DIN 1048 , es de unos $45 \mathrm{~cm}$, con un máximo de $50 \mathrm{~cm}$.

En la eInstrucción" del Ministerio de Obras Públicas se prohibe el empleo de hormigón de consistencia fluida y se aconseja una consistencia que puede llegar desde seca hasta plástica. Debe tener la docilidad necesaria para que, con los métodos previstos para la puesta en obra y compactación, el hormigón cubra las armaduras sin so- lución de continuidad y rellene completamente los encofrados sin que se produzcan coqueras. La docilidad se valorará determinando la consistencia del hormigón según uno de los dos procedimientos UNE 7102 y 7103 , recomendando para hormigones muy secos determinar su asentamiento por medio de vibrado (3).

Para disminuir la consistencia de una mezcla y hacerla más trabajable, de acuerdo con las exigencias de la obra, hay que tener muy presente que no debe aumentarse la relación $\mathrm{A} / \mathrm{C}$. Al resultar el hormigón demasiado seco, la regulación de su consistencia no debe hacerse aumentando solamente la cantidad de agua, sino al mismo tiempo la del cemento, o sea, la de la papilla de cemento, respetando la relación A/C. Otra posibilidad nos ofrece el empleo de los aditivos con el efecto plastificante-fluidificador y también el aumento del contenido en finos.

CANTIDAD DE CEMENTO. Una vez fijada la cantidad de agua necesaria y para una relación $A / C$ determinada se calcula la cantidad de cemento correspondiente. Partiendo, p. e., de la cantidad de agua $A=160$ litros y para la relación $A / C=0,60$, necesitamos la cantidad de cemento $\mathrm{C}$ :

$$
C=160 / 0,60=266 \mathrm{~kg} \text {. }
$$

El peso específico del cemento oscila normalmente entre 2,95 y 3,15 , tomando 3,10 como valor medio resulta la cantidad $\mathrm{C}$, en volumen:

$$
C=266 / 3,1=86 \text { litros. }
$$

CANTIDAD DE ARIDOS. Queda por determinar ahora la cantidad necesaria $\mathrm{Ar}$ de áridos, en volumen para completar la mezcla y obtener $1 \mathrm{~m}^{3}$ de hormigón compacto sin huecos. Hay que tener en cuenta que el hormigón ocluye también cierta cantidad $\mathrm{Ai}$ de burbujas de aire, normalmente un $1,5 \%$ del volumen total, o sea unos 15 litros/ metro cúbico. Esta cantidad aumenta al emplear aditivos con efecto aireante. En cambio podemos pres cindir del volumen de los aditivos, ya que su cantidad es insignificante:
$1 \mathrm{~m}^{3}$ de hormigón fresco se compone de:

$$
\begin{aligned}
1 \mathrm{~m}^{3}=1.000 l & =(A+C+A r+A i) l \\
1.000 l & =(160+86+A r+15) l \\
\operatorname{Ar} & =(1.000-261)=739 l .
\end{aligned}
$$

Empleando, p. e., áridos calizos cuyo peso específico es de 2,70 , necesitamos, por consiguiente, la cantidad $\mathrm{Ar}$, en peso:

$$
\mathrm{Ar}=739 \times 2,70=2.000 \mathrm{~kg} / \mathrm{m}^{3}
$$

Insistimos que se trata del peso del material seco y que su contenido en humedad se toma en cuenta para determinar la cantidad necesaria de agua de amasado.

En la técnica moderna se exige la dosificación del hormigón por peso, ya que la dosificación por volumen no determina con suficiente exactitud el contenido real de los áridos, experimentando éste cambios muy notables según su grado de humedad, estado de compactación, etc. Solamente en casos especiales se admite la dosificación por volumen, pero fijando con exactitud el peso de cada medida y comprobándolo con frecuencia, sobre todo cuando cambien las condiciones ambiente y las circunstancias del suministro del material.

\section{COMPOSICION GRANULOMETRICA} DE LOS ARIDOS. Conocemos la influencia de la composición granulométrica de los áridos sobre la calidad del hormigón $y$, según lo hemos indicado, nuestro cálculo para determinar la dosificación del hormigón impermeable está basado en el supuesto de la granulometría favorable de los áridos. Expresado en términos generales, los áridos deben ser de grano variado, con predominio del grano grueso.

Disponemos de gráficos que reseñan la composición favorable de los áridos en relación con el tamaño máximo del grano a emplear, los cuales nos facilitan la apreciación del árido disponible y, en su caso, determinar las rectificaciones necesarias. En la figura 1 está representado el área de la composición favorable delimitada por las dos curvas $A$ y $B$, para el tamaño máximo de $7 \mathrm{~mm}$ de diámetro, empleando hasta el tamaño de $0,2 \mathrm{~mm}$ un tamiz de malla y a partir de $1 \mathrm{~mm}$ tamices de paso circular. 


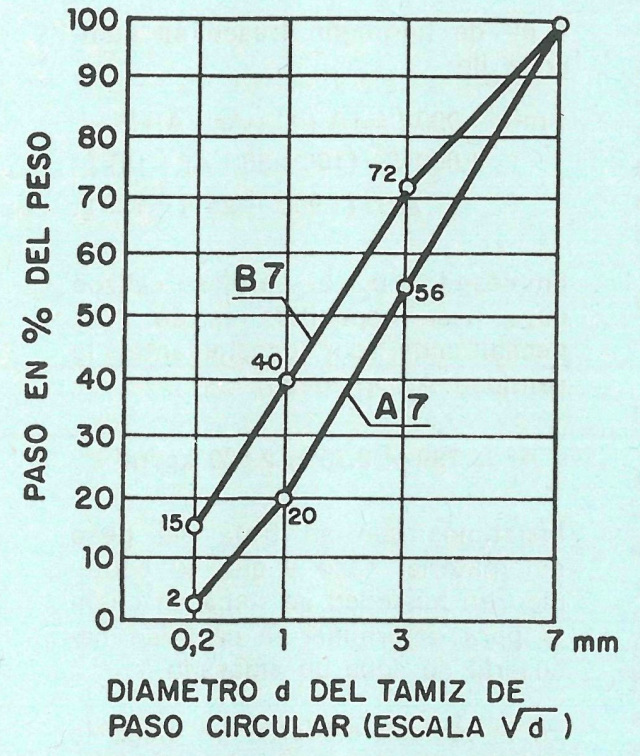

Fig. 1. Curvas límites A y B de la composición granulométrica favorable para áriposición granulo
dos de $0.7 \mathrm{~mm}$.

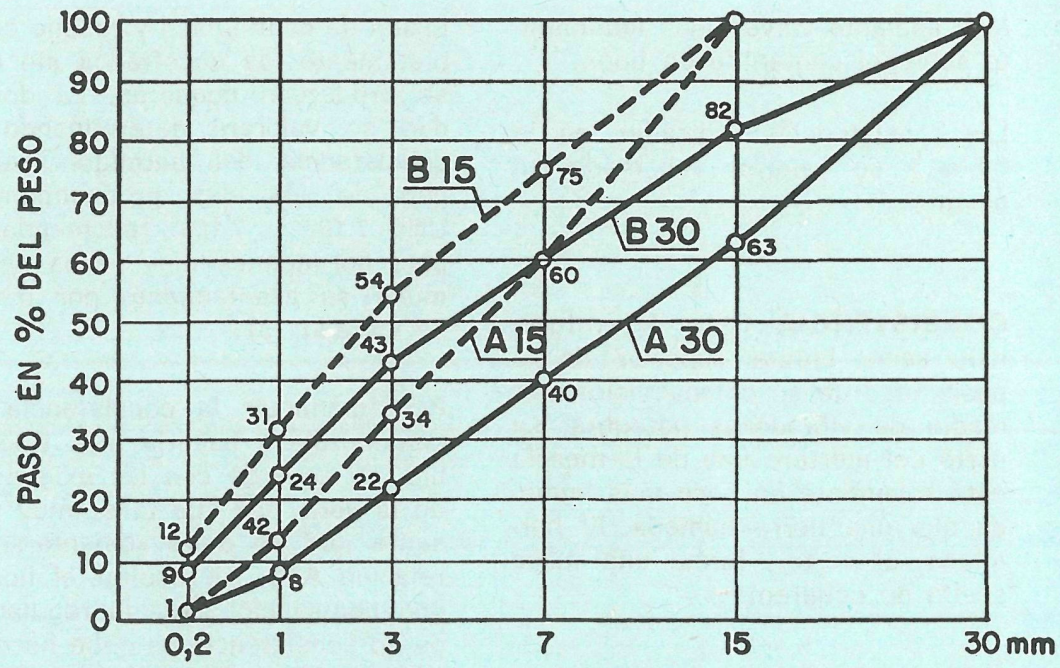

DIAMETRO d DEL TAMIZ DE PASO CIRCULAR (ESCALA $\sqrt{d}$ )

Fig. 2. Curvas límites A y B de la composición granulométrica favorable para áridos de 0.15 y de $0.30 \mathrm{~mm}$.

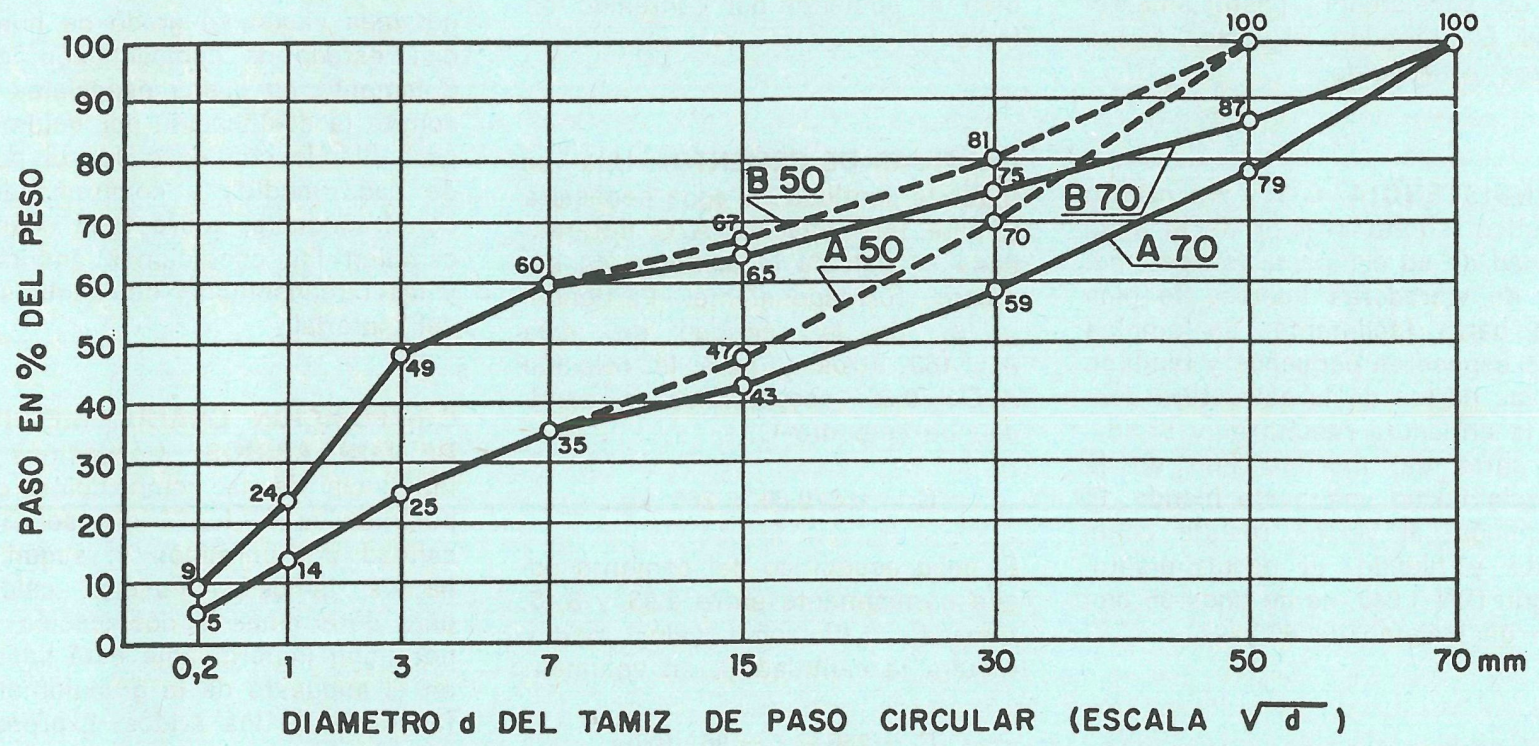

Fig. 3. Curvas límites A y B de la composición granulométrica favorable para áridos de 0.50 y de $0.70 \mathrm{~mm}$.

En la figura 2 se ven las áreas favorables para los tamaños máximos de 15 y $30 \mathrm{~mm}$, y las figura 3 nos muestra las de 50 y $70 \mathrm{~mm}$, respectivamente (2).

Para conseguir una mayor claridad de la representación se ha empleado para los diámetros la escala de $\sqrt{\mathrm{d}}$. Como se observa, estas áreas ofrecen un amplio margen para los distintos grupos de áridos dentro del árido total. Por ejemplo, para el tamaño máximo de $30 \mathrm{~mm}$ y para el grupo hasta $0,2 \mathrm{~mm}$, el margen oscila entre el 1 y $9 \%$, y para el grupo hasta $7 \mathrm{~mm}$, entre el 40 y $60 \%$.

La curva de los áridos a emplear debe situarse entre las dos curvas límites, buscando en lo posible la situación céntrica con relación a las mismas. Caso de faltar ciertos gru- pos de áridos y resultar la curva discontinua, hay que procurar que las superficies que se forman en ambos lados de la curva "ideal» sean aproximadamente iguales $y$ que la mezcla sea lo suficientemente trabajable.

Sin embargo, para un hormigón impermeable hay que exigir siempre una curva granulométrica continua y además realizar la mezcla em- 
pleando por lo menos tres distintos grupos de áridos: uno del tamaño hasta $3 \mathrm{~mm}$ y los otros dos de tamaños mayores. Se comprende que la proporción de los distintos grupos para el abastecimiento de la hormigonera debe arreglarse de tal manera que su conjunto forme una curva favorable y que la parte comprendida hasta los $7 \mathrm{~mm}$ se amolde a la representada en la figura 1.

TAMAÑO MAXIMO Y FORMA DE LOS ARIDOS. El tamaño máximo de los áridos se determina por el espesor mínimo de la estructura, siendo recomendable que no supere $1 / 5$ del mismo. Al tratarse del hormigón armado, el tamaño máximo debe ser algo inferior a la distancia mínima libre entre las armaduras (2).

Hay que cuidar también la forma de la grava, ya que su forma inadecuada puede resultar perjudicial. Según UNE 7238 , el "coeficiente" de forma $\alpha$ debe tener un valor mínimo de 0,15 , definido en la forma siguiente (3):

$$
\alpha=\frac{v_{1}+v_{2}+\ldots+v_{n}}{\frac{\pi}{6}\left(d^{3}{ }_{1}+d^{3}{ }_{2}+\ldots+d^{3}{ }_{n}\right.}
$$

en la que:

$$
\begin{aligned}
v= & \text { volumen de cada grano. } \\
d= & \text { la mayor dimensión de cada } \\
& \text { grano. }
\end{aligned}
$$

$\alpha$ mínimo $=0,15$.

CANTIDAD DE FINOS. Para obtener una buena trabajabilidad y docilidad de la mezcla, es preciso disponer de una cantidad suficiente de "finos", que actúan como una especie de lubrificante, facilitando el deslizamiento de los áridos grue- sos. Se entiende como finos el contenido de cemento conjunto con los áridos hasta $0,2 \mathrm{~mm}$.

La cantidad necesaria de finos depende del tamaño máximo de los áridos según lo indicado en la tabla 2 (2). Por ejemplo, para el tamaño máximo de $7 \mathrm{~mm}$ se necesi$\tan 500 \mathrm{~kg}$ de finos, y para $70 \mathrm{~mm}$, solamente $275 \mathrm{~kg}$. Para hormigones relativamente pobres en cemento y empleando arena muy lavada puede darse el caso de una insuficiencia en finos, requiriendo un complemento correspondiente.

Pero, por otro lado, hay que evitar el exceso de los finos, ya que éstos requieren una mayor cantidad de agua y aumentan la retracción del fraguado, perjudicando, por lo tanto, la calidad del hormigón. El empleo de los aditivos adecuados nos ofrece la posibilidad de conseguir la docilidad deseada sin tener que recurrir a un exceso de finos. Seguidamente nos vamos a ocupar de este cuarto componente del hormigón.

ADITIVOS. ¿Qué función desempeñan los aditivos en la elaboración del hormigón impermeable? Ya hemos hecho constar al principio de este informe que el empleo de un aditivo impermeabilizante por sí solo no es suficiente para asegurar la impermeabilidad del hormigón, y hemos expuesto las características necesarias que tienen que reunir el hormigón fresco y el fraguado para lograr este fin. La utilización de aditivos tiene como finalidad, por un lado, facilitar precisamente el cumplimiento de las condiciones indicadas, y por otro, corregir ciertas deficiencias del hormigón, prácticamente inevitables, aumentando además su impermeabilidad.
En una reciente publicación del au tor se trata detalladamente de las características de los aditivos para hormigones y morteros y su empleo en la construcción, por lo que nos vamos a limitar, ahora, a indicar solamente sus principales efectos (4).

HUMECTACION. Debido al efecto plastificante-fluidificador se aumenta el poder de humectación del agua de amasado, es decir, su capacidad de humedecer, lo que nos permite obtener la misma consistencia del hormigón fresco, pero empleando una menor cantidad de agua. De esta forma podemos reducir la relación $A / C$ para no sobrepasar los límites marcados para un hormigón impermeable. Además el hormigón resulta más dócil, lo que permite asegurar su buena compactación.

DISPERSION. Otro efecto muy importante de los aditivos plastificantes-fluidificadores representa su poder de dispersión. Al absorber la superficie del cemento las moléculas que forman la parte activa del aditivo se produce una carga electrostática. Las partículas del cemento quedan cargadas negativamente, repeliéndose mutuamente. por lo que se produce su dispersión y, por consiguiente, su reparto más uniforme dentro de la mezcla. Al estar las partículas de cemento mejor repartidas se aumenta la facilidad de su humectación, resultando además el hormigón más homogéneo.

HORMIGON DE CALIDAD. Por todo lo expuesto, el empleo de aditivos plastificantes-fluidificadores facilita el cumplimiento de las condi-

\begin{tabular}{|c|c|c|c|c|c|}
\hline & \multicolumn{5}{|c|}{ TAMANO MAXIMO DEL ARIDO } \\
\hline & $7 \mathrm{~mm}$ & $15 \mathrm{~mm}$ & $30 \mathrm{~mm}$ & $50 \mathrm{~mm}$ & $70 \mathrm{~mm}$ \\
\hline $\begin{array}{c}\text { Cantidad mínima en finos por } \\
1 \mathrm{~m}^{3} \text { de hormigón } \ldots \ldots \ldots \ldots\end{array}$ & $500 \mathrm{~kg}$ & $425 \mathrm{~kg}$ & $350 \mathrm{~kg}$ & $300 \mathrm{~kg}$ & $275 \mathrm{~kg}$ \\
\hline
\end{tabular}
ciones citadas para obtener un hormigón impermeable $y$, en general,

TABLA 2. Valores orientativos de la cantidad mínima en finos 
un hormigón de buena calidad. Esta es la razón del uso tan generalizado de los aditivos en los países técnicamente más avanzados. En la mayoría de los casos ya no se busca exclusivamente un efecto determinado; en cambio, lo que se pretende es mejorar en forma general las propiedades del hormigón en busca de su calidad para poder trabajar con un mayor coeficiente de seguridad. Factor éste, como sabemos, de suma importancia precisamente para el hormigón, ya que intervienen tantos factores en su preparación, colocación y curado, hasta obtener el material de características deseadas.

EXCESO DE AGUA. Sabemos que hasta en el caso más favorable y reduciendo al mínimo la relación $A / C$, para hacer la mezcla trabajable, se emplea siempre una cantidad de agua de amasado muy por encima de la cantidad necesaria para el fraguado del cemento. Para la hidratación de $100 \mathrm{~kg}$ de cemento se necesitan teóricamente sólo $18 \mathrm{~kg}$ de agua; relación $\mathrm{A} / \mathrm{C}=0,18$. Pero para convertir el cemento en una pasta manejable es preciso aumentar la cantidad de agua hasta unos $25 / 30 \mathrm{~kg}$; relación $\mathrm{A} / \mathrm{C}=0,25$ 0,30 . Como también es necesario humedecer los áridos, la relación $\mathrm{A} / \mathrm{C}$ efectiva oscila normalmente entre 0,45 y 0,70 .

En el hormigón recién colocado se encuentra, por consiguiente, siempre una cantidad muy considerable de agua que no se combina con el cemento. Este agua sobrante al evaporarse produce en el cemento fraguado una red de canales capilares intercomunicados y ramificados, debido a la presencia de granos de arena, adquiriendo la masa del hormigón una estructura microfisurada, lo que facilita la penetración del agua.

ADITIVOS IMPERMEABILIZANTES El agua de amasado que forma el tercer componente de la mezcla del hormigón representa al mismo tiempo un elemento perjudicial para su calidad, por lo que, aparte de procurar reducir en lo posible la relación $A / C$, se emplean los aditivos impermeabilizantes con el fin de corregir las deficiencias producidas por el exceso inevitable de agua. El efecto de un aditivo impermeabilizante consiste en taponar los con- ductos capilares y en rellenar los poros existentes. Conocemos la infiuencia favorable para la impermeabilidad del hormigón, sobre todo en presencia de agua a presión, al efectuar este relleno a base de materia hinchable que aumenta de volumen en contacto con el agua. De esta manera, el impermeabilizante no actúa como un hidrófugo, no repele el agua, sino que al principio absorbe una pequeña cantidad, antes de poder actuar con plena eficacia.

ADITIVOS AIREANTES. Los aditivos con efecto aireante actúan también favorablemente sobre la impermeabilidad del hormigón al ocluir dentro de su estructura aire adicional en forma de burbujas minúsculas, esferoides, de 50-300 $\mu$, estables y uniformemente repartidas. Estas burbujas de aire interrumpen la continuidad de conductos capilares, por lo que se obstaculiza la penetración de agua dentro de la masa del hormigón, resultando éste más impermeable y más resistente contra los efectos de las heladas.

RESISTENCIA DE HORMIGON. Una de las primeras condiciones que se exigen al emplear un aditivo, es que no debe disminuir la resistencia del hormigón. Solamente en casos determinados, y salvo otro remedio, se admite una cierta reducción de la resistencia.

Sabemos, p. e., que la oclusión de aire adicional disminuye la resistencia del hormigón a la compresión. A título de información, indicamos que para cada un $1 \%$ de aire ocluido hay que contar con una disminución de la resistencia a la compresión de un 3-4\%. Ahora bien, debido al efecto plastificante de las burbujas de aire que actúan de hecho como una especie de rodamiento de bolas, facilitando el deslizamiento de los áridos, se necesita menos agua para hacer el hormigón trabajable y tenemos la posibilidad de reducir la relación $\mathrm{A} / \mathrm{C}$, lo que compensa, por lo menos en gran parte, la influencia desfavorable de la oclusión de aire adicional.

En unos ensayos realizados con un aditivo impermeabilizante a base de materias albuminoides, de pronunciado efecto plastificante-fluidificador, se observaron aumentos de resistencia a la compresión en 12,5 y $18,0 \%$, a pesar de haber empleado una cantidad exagerada del aditivo y haber subido el aire ocluido a 8,7 y $9,1 \%$ (5). Esta notable me jora de la resistencia era debida a la posibilidad de reducir la relación $\mathrm{A} / \mathrm{C}$ desde 0,795 hasta 0,615 y desde 0,760 hasta 0,610 , respectivamente, pero conservando, lo que es importante, la misma trabajabilidad del hormigón.

Pero como, por otro lado, se observa con frecuencia una disminución de la resistencia al añadir aditivos impermeabilizantes, se recomienda una selección adecuada de los mismos, insistiendo sobre la importancia del efecto plastificantefluidificador.

AMASADA DE ENSAYO. Una vez determinadas las características y las cantidades de los cuatro componentes del hormigón es conveniente amasar, en plan de ensayo, una cantidad de unos 50 litros para comprobar si el material resultante reúne las cualidades exigidas, o si fuera necesario, efectuar las rectificaciones correspondientes.

Si la papilla de cemento empleada resultara insuficiente para rellenar los huecos del hormigón compactado, habría que aumentar su cantidad. Al resultar la consistencia del hormigón demasiado seca o demasiado blanda para la trabajabilidad deseada, habría que aumentar o disminuir, respectivamente, la proporción del aditivo plastificante o de la cantidad de la papilla de cemento, pero teniendo el cuidado de no aumentar la relación $\mathrm{A} / \mathrm{C}$ y de no rebajar la cantidad mínima admisible de cemento de $250 \mathrm{~kg} / \mathrm{m}^{3}$.

También hay que comprobar, por medio de ensayos correspondientes, si el hormigón obtenido cumple las condiciones necesarias de resistencia y de impermeabilidad, antes de aceptar para su ejecución la composición prevista del mismo.

ASPECTO DE LA GARANTIA. ¿Cómo se presenta el aspecto de la garantía en las obras realizadas a base de hormigón impermeable? ¿Son garantizables esta clase de obras?

CONDICIONES TECNICAS. Para aclarar este concepto tenemos que tener presente que la composición 
adecuada del hormigón forma solamente uno de los elementos de la obra acabada y debe complementarse con la preparación y puesta en obra correctas. El amasado debe efectuarse cumpliendo estrictamente las condiciones fijadas para la dosificación y características de los distintos componentes del hormigón. Hay que evitar que se produzca una disgregación del mismo durante su transporte y colocación, debiendo llegar con la trabajabilidad adecuada para asegurar su buena compactación y no acusar un principio de fraguado.

No se colocarán capas o tongadas con espesor mayor al que permite la compactación completa de la masa.

El problema de las juntas de trabajo debe estar satisfactoriamente resuelto y su unión efectuada con el máximo cuidado. Finalmente hay que cuidar del adecuado curado del hormigón.

El acabado del hormigón impermeable debe efectuarse normalmente con un revoco igualmente impermeable.

Se presupone naturalmente que el proyecto de la obra debe ser correcto, lo mismo referente al cálculo de la resistencia que a la ordenación de los distintos elementos estructurales, a las juntas de dilatación, etc.

POSIBILIDAD DE FALlOS. Cumpliendo las condiciones citadas estamos razonablemente autorizados a suponer que se va a obtener un hormigón de características requeridas, o sea, un hormigón impermeable y la estanqueidad de la obra. Sin embargo, hay que contar siempre con la posibilidad, más o menos remota, de algún fallo, sea en la calidad del material, sea en la ejecución, o por falta de resistencia mecánica al resultar la presión efectiva de agua más elevada de la prevista, debido a la subida anormal del nivel freático, a las condiciones climatológicas excepcionales, o a otras causas.

La ejecución de la obra a ritmo acelerado $o$ en condiciones climatológicas adversas puede ser la causa de ciertas deficiencias sin importancia para la construcción en general, pero de consecuencias graves para la impermeabilización, ya que el agua acusa normalmente cualquier fallo, por muy pequeño que sea. Una fisura insignificante puede provocar, en ciertas circunstancias, el vaciado de un depósito o la inundación de una construcción subterránea.

También debemos, naturalmente, no olvidar que la impermeabilización a base de un hormigón impermeable tiene las características de una impermeabilización rígida, que no actúa como una impermeabilización elástica, lo que significa que al producirse un agrietamiento de la estructura queda también afectada la eficacia de la impermeabilización.

No se pretende, como es natural, resaltar los peligros de los fallos, sino hacer constar solamente la posibilidad de éstos, para que el técnico responsable de la obra aprecie el riesgo que puedan representar. y actuar en consecuencia.

En una obra bien llevada y cumpliendo las condiciones objeto de esta publicación es muy difícil que se produzcan fallos de impermeabilización generalizados; pueden ocurrir defectos locales, cuyo remedio es relativamente fácil.

Además, y como medida de precaución, el hormigón impermeable se completa normalmente con un revoco también impermeable, y tratándose de depósitos, con una pintura impermeable.

POSIBILIDAD DE GARANTIA. Sin embargo, cada obra, sin excepción, representa indiscutiblemente un cierto riesgo, por muy pequeña que sea la probabilidad de que ocurra un siniestro. ¿Existe un procedimiento para cubrir este riesgo cuando se trata de una impermeabiliza ción a base de un hormigón impermeable?

Un requisito indispensable para asegurar el resultado de la impermeabilización es un control eficaz y permanente de la ejecución de la obra de acuerdo con las condiciones tratadas anteriormente.

Se entiende, por lo expuesto, que no es factible exigir del suministrador del aditivo impermeabilizante una garantía de la eficacia de la impermeabilización. Su intervención se limita solamente a facilitar uno de los componentes del hormigón $y$ a las indicaciones relativas a su empleo, pero no tiene prácticamente ninguna intervención en la ejecución de la obra. El importe de los aditivos representa un capítulo de escasa importancia económica para el conjunto de la obra. Sin embargo, un fallo de la impermeabilización puede producir perjuicios económicos sumamente elevados, sobre todo en las construcciones subterráneas con presencia de agua a presión, al estropearse la mercancía almacenada o al averiarse las conducciones eléctricas, las instalaciones de calefacción, de aire acondicionado, etc.

Existe una desproporción demasiado elevada entre estos perjuicios y el importe de los aditivos para que su suministrador pueda actuar como su propio asegurador. Por cierto, una rotura de una tubería de agua o un desbordamiento de una alcantarilla puede provocar la inundación del subterráneo y producir daños de la misma indole (6).

Lo único que se exige del suministrador de los aditivos en los países técnicamente avanzados, p. e., en Alemania, es el cumplimiento del contrato de suministro, es decir, que el material suministrado corresponda al material ofrecido. Como medida de precaución se deposita una muestra del mismo para poder hacer la comprobación correspondiente en caso de surgir alguna duda.

Cuando interesa poder contar con una garantía efectiva y no basada en el factor suerte, aparte de cumplir estrictamente las condiciones técnicas expuestas, la intervención de una Compañía de Seguros en colaboración con una Entidad de Control de Obras parece indicar un camino para solucionar satisfactoriamente este problema.

Este procedimiento se emplea con cierta frecuencia en Francia, pero tenemos entendido que se exige normalmente el Seguro de la totalidad de la obra de fábrica para que las primas no resulten prohibitivas.

CONCLUSION. Al proyectar una obra de impermeabilización a base de un hormigón impermeable y sa- 
biendo que el empleo de un aditivo impermeabilizante por sí solo no es suficiente para asegurar su estanqueidad, el hormigón debe reunir ciertas características de trabajabilidad y de homogeneidad, sin que la relación $\mathrm{A} / \mathrm{C}$ y la cantidad de cemento por metro cúbico rebasen un determinado valor.

Para el cumplimiento de estas condiciones hay que fijar la dosificación del hormigón de acuerdo con la interrelación que existe entre sus distintos componentes, partiendo de una favorable composición granulométrica de los áridos y de

\section{BIBLIOGRAFIA}

(1) ¿Vorläufige Richtlinien für die Prüfung von Betonzusatzmitteln zur Erteilung von Prüfzeichen», Fassung Januar 1965.

(2) Prof. Dr. Ing. Kurt Walz: "Anleitung für die Zusammensetzung und Herstellung von $\mathrm{Be}-$ ton mit bestimmten Eigenschaften», Berlin-München, 1963. la necesaria cantidad de agua de amasado.

Debido al empleo de los aditivos adecuados, que forman el cuarto componente del hormigón, se facilita el cumplimiento de las citadas condiciones y se aumenta su impermeabilidad.

Aparte de la dosificación del hormigón, es de suma importancia que su preparación, su puesta en obra y su curado final se ejecuten en forma adecuada, por lo que es preciso poder contar con un control de obras eficaz.

(3) "Instrucción para el proyecto y la ejecución de obras de hormigón en masa o armado , Ministerio de Obras Públicas, 1968.

(4) C. Safránez: “Características de los principales aditivos químicos para hormigones y morteros y su empleo en la construcción", "Informes de la Construcción», núm. 224, octubre 1970.
Cumpliendo lo expuesto, estamos autorizados a esperar la obtención de un hormigón impermeable y la estanqueidad de la construcción.

Sin embargo, hay que tener presente que todas las obras represen$\tan$ un cierto riesgo $\mathrm{y}$, dada la complejidad de una obra a base de hormigón impermeable, su garantía presenta un problema de difícil solución. Donde interesa poder contar con una garantía realmente eficaz, un camino prometedor representa la intervención de una Compañía de Seguros en colaboración con una Entidad de Control de Obras.

(5) C. Safránez: "Aditivos impermeabilizantes a base de derivados de materias albuminoides y su efecto en hormigones y morteros", "Cercha", núm. 6 , año IV.

(6) C. Safránez: «La garantía en las obras de impermeabilización", "Temas de Arquitectura y Urbanismo", núm. 126, diciembre 1969.

\section{résumé}

Considérations sur le béton imperméable et l'aspect de sa garantie

Carlos Safránez, Dr. ingénieur

L'auteur définit les conditions à remplir par le béton frais et la prise pour donner lieu à un béton imperméable. II indique le procédé à suivre afin de déterminer son procédé à suivre afin de déterminer son dosage en accord avec I'interrélation exispartir de la quantité nécessaire d'eau de gâchage en rapport avec la consistance des agrégats.

II explique I'action des adjuvants plastifiants-fluidifiants, entraîneurs d'air et produits d'étanchéité, qui forment le cadre composant du béton, dont les effets permettent de remplir les conditions nécessaires et de corriger certains défauts inévitables.

En outre, l'auteur insiste sur l'importance qu'a un contrôle effectif de l'exécution de l'ouvrage, particulièrement en ce qui conen oeuvre et de sa cure.

Finalement, il expose l'aspect de la garantie, problème d'une certaine complexité, et indique le chemin à suivre pour trouver une solution satisfaisante.

\section{summary}

Notes on watertight concrete and on its quality control

Carlos Safránez, Dr. engineer

Conditions to be met by fresh concrete, including its setting process, to obtain wroportioning method, in terms of the proportioning method, in terms of the defined in terms of the necessary mix water to obtain a given consistency, and in relation to the aggregate proportioning.

The action of the plastifying, fluidifying air entraining and waterproofing additives is described. These additives constitute the fourth component of concrete. Their influence adds the above mentioned properties to of this medium.

The author also emphasizes the need to control carefully the placing of the concrete at the site, as well as the mixing process equally important, and should be precisely supervised.

Reference is also made to the question of guaranteeing the quality of the concrete, when treated with waterproofing additives. This involves certain difficulties, and the author indicates possible ways of overcoining them.

\section{zusammenfassung}

Betrachtungen über undurchlässigen Beton und den Aspekt seiner garantie

Dr. Ingenieur Carlos Safránez

Der Autor definiert hier die Bedingungen, die an den frischen und abgebundenen Beton gesteltt werden müssen, um einen undurchlässigen Beton zu erzielen. Er beschjeweiligen Dosierung entsprechend des bestehenden Zusammenhangs zwischen den verschiedenen Komponenten, ausgehen von der erforderlichen Mischwassermenge im Hinblick auf die gewünschte Konsistenz und granulometrische Zusammensetzung der Zuschlagstoffe.

Er erläutert die Wirkung der Betonverflüssiger, LP-Stoffe und Dichtungsmittel als vierter Komponente des Betons, mit denen die erwähnten Bedingungen erfüllt und gewisse unvermeidliche Mängel behoben werden können.

Der Autor besteht auf der Notwendigkeit einer effektiven Kontrolle der Ausführung, insbesondere hinsichtlich der Betonzubereitung, seiner Aufbringung und Nachbehand lung.

Abschliessend wird das recht komplexe Problem der Garantie untersucht und de Weg auf der Suche nach einer zufriedenstellenden Lösung gewiesen. 